\title{
CLAVE DE IDENTIFICACION PARA GENEROS DE BIVALVOS AMAZONICOS (MOLLUSCA : UNIONOIDA) EN EL PERU
}

\author{
KARINA VALDIVIA $P^{2,4}$ \\ RONALD VIVAR $G,{ }^{1,4}$ \\ PEDRO HUAMÁN M. ${ }^{2,4}$ \\ JAVIER ZALDIVAR R. ${ }^{3}$
}

1. Departamento de Malacol.gía y Carcinología. Museo de Historia Natural Apdo. 140434. Lima 14 - Perú.

2.- Labc zatorio de Fauna Dulceacuícola. Instituto de Investigación "Antonio Raimondi". Facultad de Ciencias Biológicas. Universidad Nacional Mayor de San Marcos. Apdo, 10235. ILima

3.- IVITA. Daniel A. Carrión 319. Pucallpa.

4.- Laboratorio de Invertebrados. Fac. de Ciencias Biológicas. Universidad Ricardo Palma. Av. Benavides cda. $54 \mathrm{~s} / \mathrm{n}$. Fax. 449-2477. Lima.

\section{RESUMEN}

Se presenta una clave de identificación para géneros de bivalvos amazónicos basada en caracteres de las partes blandas y de la concha. Se indican detalles de seis géneros (Castalia, Diplodon, Mycetopoda, Anodontites, Leyla y Bartlettia).

PALABRAS CLAVES: Bivalvia, taxonomía, Unionoida, morfología, Etheriidae.

\section{SUMMARY}

A key of identification for genera of amazon bivalvia based upon the characters of the shell and soft parts is presented. Shell and anatomic detailed for six genera (Castalia, Diplodon. Mycetopoda, Anodontites, Leyla and Bartlettia) are indicated.

KEY WORDS: Bivalvia, taxonomy, Unionoida, morphology, Etheriidae.

\section{INTRODUCCION.}

Los unionoidos generalmente llamados "náyades" tienen interés por ser recursos potenciales de la dieta humana y gozan de alta demanda en la explotación de nácar con fines artesanales (Castellanos y Landoni 1990); además son importantes desde el punto de vista ecológico, por ser indicadores paleoambientales (Warren, 1991; 1995), así como también de la calidad del agua (Strayer y Smith), 1993).

Desde el siglo pasado, numerosas descripciones de especies, han sido establecidas para la región Neotropical en base al estudio de la morfología externa. Pero se presentan dificultades taxonómicas al caracterizar al especies y géneros por la variabilidad intra, interpoblacional y específica (Parodiz, 1982), producida por la transición de cuerpos dulceacuícolas y su distribución heterogénea debido a la fijación de los estadíos larvales en estructuras de peces límnicos de amplia distribución sudamericana (Castellanos y Landoni, op. cit. ). La solución del problema se basa actualmente en el análisis detallado de la morfología del sistema digestivo y descriptivo de otros sistemas de órganos; además de estructuras especiales de las formas larvales que aportan caracteres para diferenciar los taxa genéricos y específicos. Se han realizado estudios anatómicos de bivalvos sudamericanos por Mansur (1972,1974); Veintenheimer y Mansur , (1978).

En el Perú, el estudio de los bivalvos de agua dulce no ha recibido atención durante décadas, solo un estudio preliminar de moluscos amazónicos nos indica la presencia de algunos géneros (Mycetopoda, Bartlettia, Anodontites y Diplodon) (Vivar et al ,1992), en el que se sugiere el estudio anatómico de las partes blandas para caracterizarlos correctamente. Por tal motivo, la presente comunicación contiene una clave basada en aspectos morfológicos de la anatomía y de la concha, constituyendo un aporte para el conocimiento de la malacofauna en nuestro país.

\section{MATERIAL Y MÉTODOS}

Los bivalvos examinados fueron colectados durante los meses de Julio-Agosto 1991, Junio 1992, Abril-Mayo 1994 
y Febrero 1995, en algunas localidades de Pucallpa (Ucayali), Huánuco, Satipo (Junín ) y el Manú (Madre de Dios).

Los especímenes fueron colocados en alcohol al 70 $\%$ y en formol al $4 \%$ para preservar las partes blandas siguiendo a Mansur y Oliveira (1990). Estructuras de importancia taxonómica se presentan en las figuras 1 y 2 . Para el examen detallado se utilizó material obtenido por microdisección; las observaciones se efectuaron en un microscopio estereoscopio CARL ZEISS. Las fotografías se obtuvieron con una cámara NIKON. En las figuras 19, las barras de las escalas representan $1 \mathrm{~cm}$.

Para la preparación de la clave también se utilizó información bibliográfica referida a bivalvos sudamericanos. El material colectado forma parte de la colección de moluscos del Laboratorio de Fauna Dulceacuícola $\left(\mathrm{N}^{\circ} 216\right)$ de la Facultad de Ciencias Biológicas de la U.N.M.S.M.

\section{RESULTADOS}

En base al estudio morfológico de la concha y de las partes blandas se preparó las siguiente clave:

\section{CLAVE DE IDENTIFICACION DE ALGUNOS GENEROS DE BIVALVOS AMAZONICOS PERUANOS}

1a. Conchilla única, rádula, manto diferenciado en branquias y/o cavidad pulmonar, terrestres y acuáticos

1b. Dos valvas, ausencia de rádula,manto simple, branquias lamelares; acuáticos con charnela edentula o dentada.....

2a. Equivalvos, o inequivalvos generalmente con dientes en la charnela, los dos laterales alargados (no separados de los cardinales), algunos disodontos. Capa nacarada

2b. Equivalvos, pocos dientes cardinales, gruesos, separados de los dientes laterales, concha sin capa nacarada, Heterodontos.

3a.De medianas a grandes alargados en sentido antero-posterior elípticas, ligeras; dulceacuícolas

3b.Pequeñas a medianas semiesféricas, delgadas; usualmente equivalvas e isomiarios. La mayoría marinos

4a. Conchilla sin escultura umbonal; charnela con o sin dientes, impresión del músculo aductor unido al retractor; Abertura branquial con papilas o tentáculos distribuídos en forma ordenada; esófago corto achatado, con o sin anillo circular. Intestino con cuatro vueltas y tres regiones distinguibles. Abertura supranal presente. Marsupio en casi toda la demibranquia interna. Larva lasidio.

4b. Conchilla de aspecto foliar, periostraco de fácil desprendimiento; charnela sin dientes. Pie reducido o ausente; abertura anal amplia; isomiarios, dimiariosLarva lasidio.

4c. Conchilla con escultura umbonal. Charnela con dientes; impresión del músculo aductor separado del retractor. Abertura branquial con tentáculos dispuestos en forma desordenada; esófago estrecho sin canal circular; intestino con dos vueltas. Sin abertura supranal. Marsupio en posición central. Larva gloquídea.

5a.Conchilla subtrapezoidal, alargada, poco inflada, umbos bajos , cavidad subumbonal reducida. Charnela larga, edentula. Margen dorsal
Clase Gastropoda

Clase Bivalvia (2)

SubClase Palaeoheterodonta (3a)

Sub Clase Heterodonta (3b)

Orden Unionoida (4)

Orden Veneroida

Familia Mycetopodidae (5)

Familia Etheriidae (6a)

Familia Hyriidae (7) 
horizontal. Capa prismática estrecha o ausente. Cresta radial poco elevada, declive posterior marcado. Impresiones musculares anteriores más pequeñas que las posteriores.Abertura branquial en forma de sifón papiloso. Palpos labiales largos, redondeados. Marsupio en la mitad anterior de la demibranquia interna.

5b. Conchilla redondeada-elíptica. De leve a inflada, abertura intervalvar. Umbos variables. Margen dorsal variable. Capa prismática gruesa y ancha. Cresta radial y declive posterior de leve a bien definido. Impresiones musculares notorias. Abertura branquial con o sin papilas. Palpos labiales semicirculares. Marsupio en casi toda la demibranquia interna.

5c. Conchilla grande con proyecciones alares; impresiones musculares dorsales, sin abertura intervalvar. Charnela con alguna nodosidad. Seno paleal marcado. Sifones desarrollados, retráctiles. Abertura branquial y exhalante con tentáculos. Palpos labiales son alargados. Marsupio grande ocupando casi toda la demibranquia interna.

6a. Con fuerte deflexión ventral; con reduccióndel músculo protractor y retractor; pie vestigial; palpos labiales también reducidos; abertura branquial con tentáculos bien diferenciados; Marsupio ocupa casi toda la demibranquia interna exepto los extremos.

7b. Inflados.Carinación fuerte en el lado posterior, escultura umbonal con estrías radiales. Abertura branquial inferiormente fusionada; con numerosos tentáculos. Palpos labiales semicirculares. Expansión lateral del estómago reducida. Ano generalmente con membranas rectangulares Gloquídeo sin flagelo adhesivo y desarrollo indirecto

7c. Poco inflados, estrías convergentes. Carinación poca profunda. Abertura branquial inferiormente libre, con pocos tentáculos. Expansión lateral del estómago desarrollada. Ano sin membranas rectangulares. Gloquideo con flagelo adhesivo y desarrollo directo o indirecto.
Género Mycetopoda Orbigny, 1835

Género Anodontites Bruguiére, 1792

Género Leyla Gray, 1840

Género Bartlettia Moricand, 1856

Género Castalia Lamarck, 1819

Género Diplodon Spix, 1827
Las figuras de algunas especies de bivalvos (Figs: 3-9), son una muestra representativa de los géneros que ocurren en nuestro territorio. Algunas de éstas figuras constituyen por primera vez un documento gráfico de nuestra malacotaxa de bivalvos.

\section{COMENTARIOS}

Los bivalvos sudamericanos están representados por tres superfamilias, Unionoidea(contiene a la familia Hyriidae), Muteloidea (agrupa los Etheriidae y Mycetopodidae) y Sphaerioidea (Sphaeridae y Corbiculidae). Las familias de los dos primeros grupos son revisadas en la presente comunicación en base al estudio de algunos géneros que se presentan en diferentes localidades del territorio peruano.

La familia Hyriidae está representada por los géneros Castalia Lamarck, 1819, Diplodon Spix, 1827, Triplodon Spix, 1827, Callonaia Simpson, 1900 y otros que han sido registrados como material fósil (Ver Parodiz, 1968).
La semejanza conquiológica se puede mostrar en las figuras 3 y 4 para los géneros Castalia y Diplodon ocasionando dificultades para separarlos debido a que poblaciones de diversas especies de éstos géneros habitan algunos cuerpos de agua en forma simultánea (e.g. Ucayali). Las diferencias anatómicas se pueden evidenciar a nivel de la fusión de la abertura branquial, detalles del sistema digestivo y otros relacionados a la larva gloquideal. La distribución geográfica de Castalia incluye casi la totalidad de la región sudamericana, exceptuando las zonas occidentales de Ecuador, Perú y Chile (Bonetto, 1965); demostrando que este género y probablemente otros presentan una gran plasticidad ecológica que dificulta la identificación de las especies, debido a las variaciones que se observan cuando especies emparentadas tienen la posibilidad de cruce al ocurrir en el mismo habitat (hibridización). Diplodon es el género que ha sido estudiado ampliamente en América Tropical. Parodiz (1982), considera que sólo 60 especies son válidas de un total de 300 nombres específicos que han sido descritos en numerosas 
monografías genéricas y revisiones de carácter específico; y es probable que el número sea menor, porque esta malacotaxa presenta una marcada variabilidad (a nivel de género y especie), y sería un ejemplo ilustrativo de probables fenómenos evolutivos de especiación.

Los otros géneros de Hyriidae (no considerados en éste trabajo), presentes en el Perú (Triplodon, y otros) tienen representantes, pero sólo contamos con material conquiológico y no con partes blandas que nos permitan dilucidar algunos problemas específicos o de tipo nomenclatorial.

La familia Etheriidae comúnmente llamadas «ostras de agua dulce», presentan en su compocisión genérica a cuatro grupos: Etheria Lamarck, 1807; Pseudomulleria Anthony, 1905; Acostea Orbigny, 1851 y Bartlettia Adams, 1866 , caracterizados por su concha de aspeco foliar, no firme, periostraco de fácil despredimiento, charnela edentula, pie reducido y una abertura anal amplia (Mansur y Oliveira, 1990).

En América del Sur, Acostea y Bartlettia tienen representantes; a pesar de no presentar semejanzas en la concha, ambas comparten caracteres durante su crecimiento y desarrollo, donde se expresa un alargamiento ántero-posterior con extensión dorsoventral de la zona posterior y reducción de la zona anterior en el adulto (Mansur y Oliveira op.cit., fig.13).

Bartlettia stefanensis (probablemente la única especie conocida) fue tentativamente considerada como especie polimorfa de Anodontites tenebricosus por la semejanza entre los estadíos juveniles de Bartlettia y los adultos de Anodontites; incluso por su posición en el habitat (sustrato endurecido) donde presentan una deflexión ventral leve (similar a algunos géneros de Mycetopodidae). Estas consideraciones y otros detalles probablemente permitan explicar la relación filogenética de los Etheriidae con los Mycetopodidae. Mayor información taxonómica (anatomía de las partes blandas), biológica (reproductiva y desarrollo) y zoogeográfica (distribución) de las especies neotropicales permitirán reforzar la conexión filogenética que involucra a los Etheriidae dentro de la superfamilia Muteloidea tal como lo muestra Mansur y Oliveira op.cit.

La familia Mycetopodidae compuesta genéricamente por Mycetopoda Orbigny, 1835; Anodontites Bruguiere,1792; Leyla Gray,1840, Monocondylaea Orbigny, 1835; se distribuyen en América del Sur en toda la cuenca Amazónica y concentra un buen número de especies en la zona del Paraná-Paraguay-Uruguay (Bonetto,1965). Se observan pequeñas diferencias anatómicas entre los géneros de esta familia, considerando músculos aductores y retractores, branquias, marsupio, palpos labiales y masa visceral.
Mansur y Valer (!992), presentan un ordenamiento taxonómico donde incluyen a la familia Mycetopodidae en la superfamilia Unionoidea, probablemente por una omisión de la superfamilia Muteloidea después de mecionar las especies de la Tribu Prisodontini de los Hyriidae.

Como se mencionó anteriormente para B. stefanensis, sus estadíos juveniles son símiles a varias especies adultas de Mycetopodidae, por la presencia de marsupio en la demibranquia interna, canal alimenticio (esófago, estómago e intestino), palpos labiales grandes, branquias y abertura supranal. Es evidente que los caracteres que diagnostiquen a las especies peruanas de Mycetopodidae aún no determinadas y las de Etheriidae, limitan la interpretación de las relaciones filogenéticas entre ambos grupos. Un alcanze mayor en las prospecciones en la región amazónica permitirá establecer un inventario de la diversidad malacológica y facilitará la interpretación correcta sobre los patrones de distribución geográfica de estos grupos.

\section{AGRADECIMIENTOS}

Parte de este trabajo fue subvencionado por el FEDU (UNMSM) (Proyecto $N^{\circ}$ 4100127). A la Dra. Maria Cristina D. Mansur de la Fundacão Zoobotánica do Rio Grande do Sul (Brasil) por su apoyo bibliográfico. Al Blgo. Jésus Córdova Santa-Gadea por su apoyo logístico.

$\begin{array}{ll}\text { ABREVIATURAS } \\ \text { a } & \text {-ano } \\ \text { ae } & \text {-abertura exhalante } \\ \text { ai } & \text {-abertura inhalante } \\ \text { as } & \text {-abertura supraanal } \\ \text { bp } & \text {-borde prismático } \\ \text { ch } & \text {-charnela } \\ \text { cp } & \text {-capa prismática } \\ \text { csu } & \text {-cavidad sub-umbonal } \\ \text { d } & \text {-diafragma } \\ \text { dbe } & \text {-demibranquia externa } \\ \text { dbi } & \text {-demibranquia interna } \\ \text { ima } & \text {-impresiones musculares anteriores } \\ \text { imp } & \text {-impresiones musculares posteriores } \\ \text { l } & \text {-línea paleal } \\ \text { li } & \text {-ligamento } \\ \text { m } & \text {-manto } \\ \text { maa } & \text {-músculo aductor anterior } \\ \text { map } & \text {-músculo aductor posterior } \\ & \end{array}$




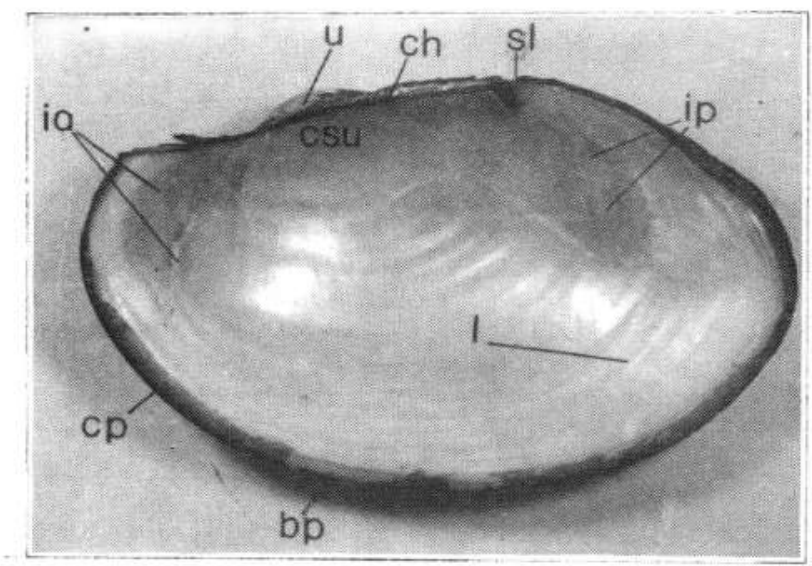

Fig. 1: Cara interna de la valva izquierda de Anodontites trapesialis, con algunos detalles de la concha.

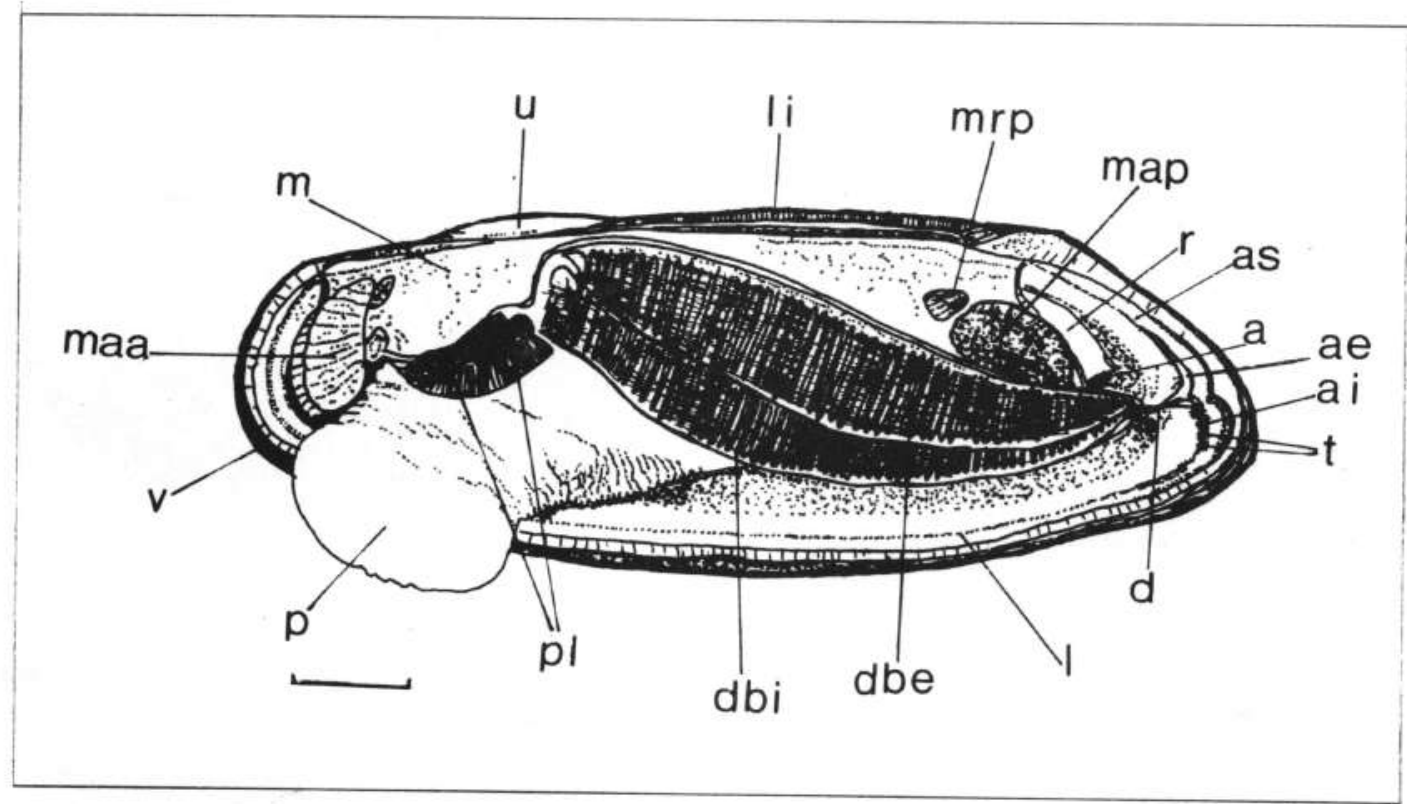

Fig. 2: Morfología interna de Mycetopoda legumen, (Martens, 1888).
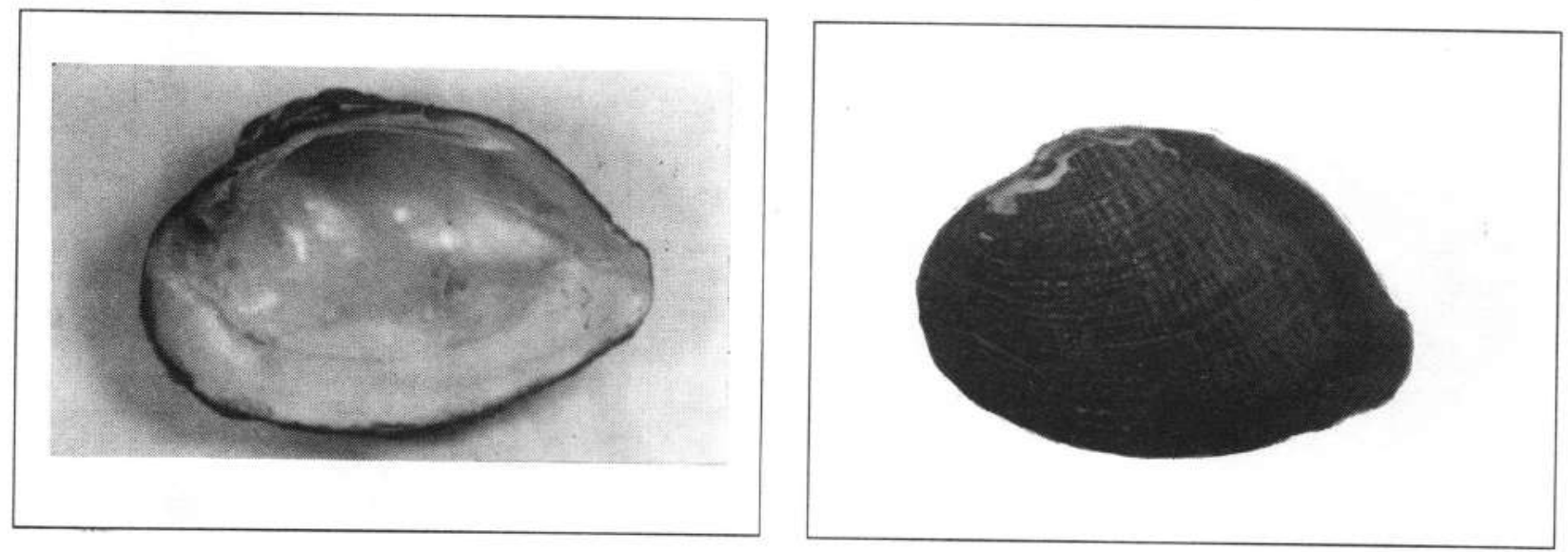

Fig.3: Concha de Castalia ambigua,(Lamarck, 1819). Izquierda: vista interna, Derecha: vista externa. 


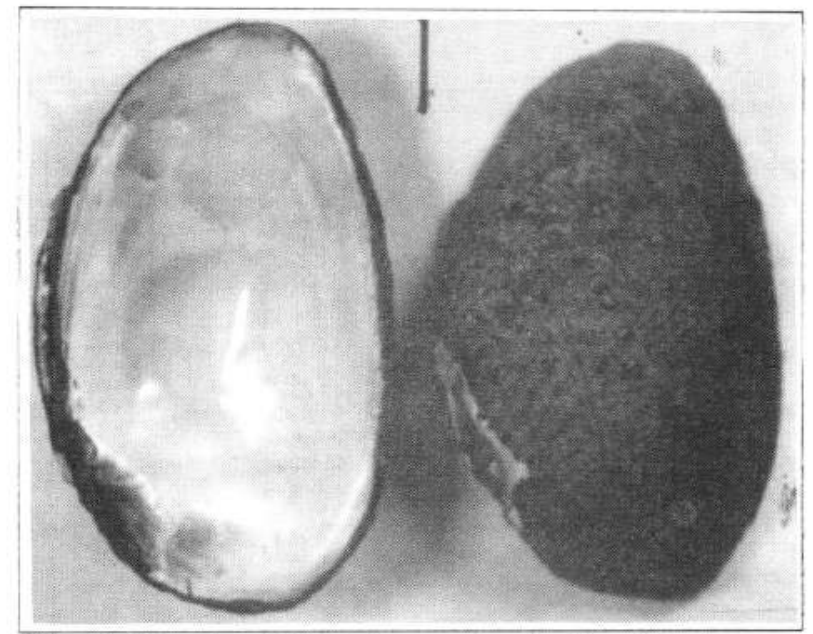

Fig. 4: Concha de Diplodon sp. Izquierda: vista interna. Derecha: vista externa.

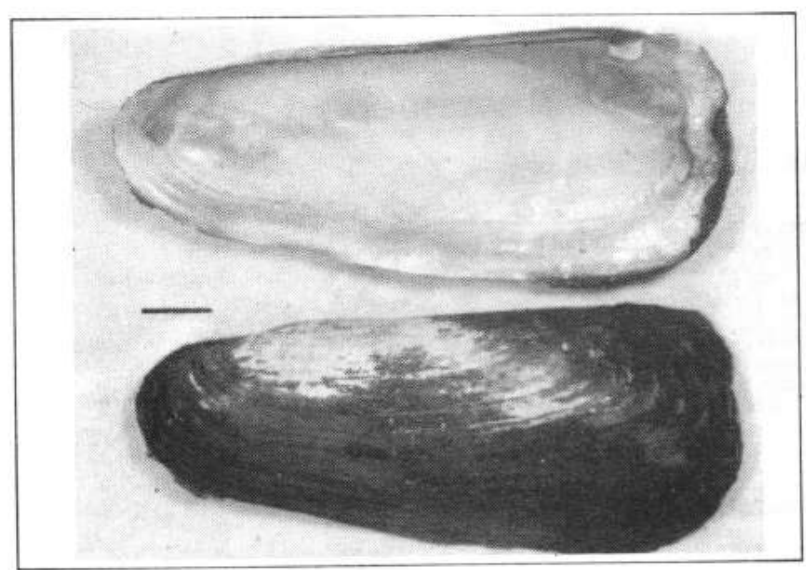

Fig. 6: Concha de Mycetopoda siliquosa, (Spix, 1827) Superior: vista interna. Inferior: vista externa.

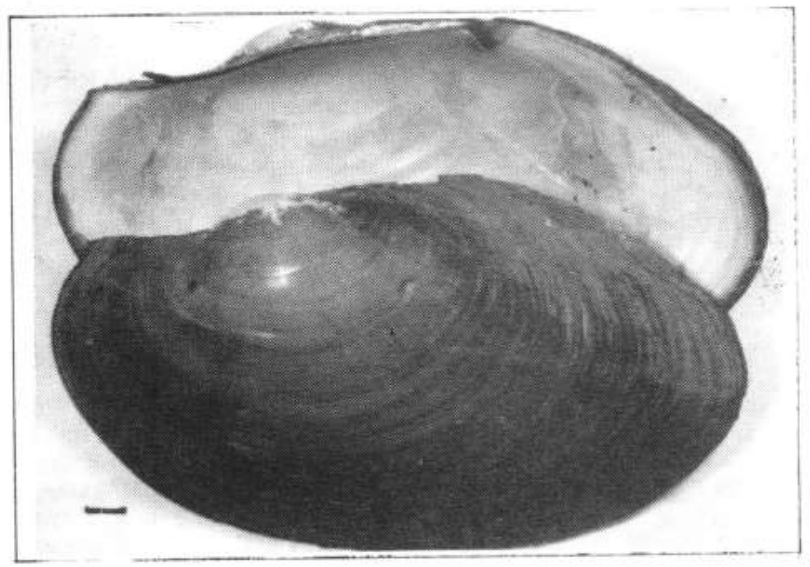

Fig. 8: Concha de Anodontites trapesialis, (Lamarck, 1819) Superior: vista interna. Inferior: vista externa.

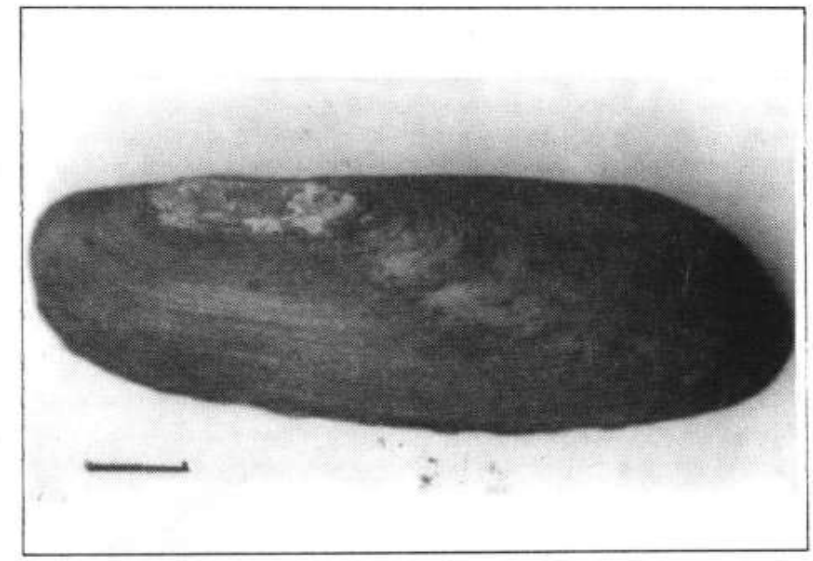

Fig. 5: Concha de Mycetopoda legumen, (Martens, 1888) Vista externa.

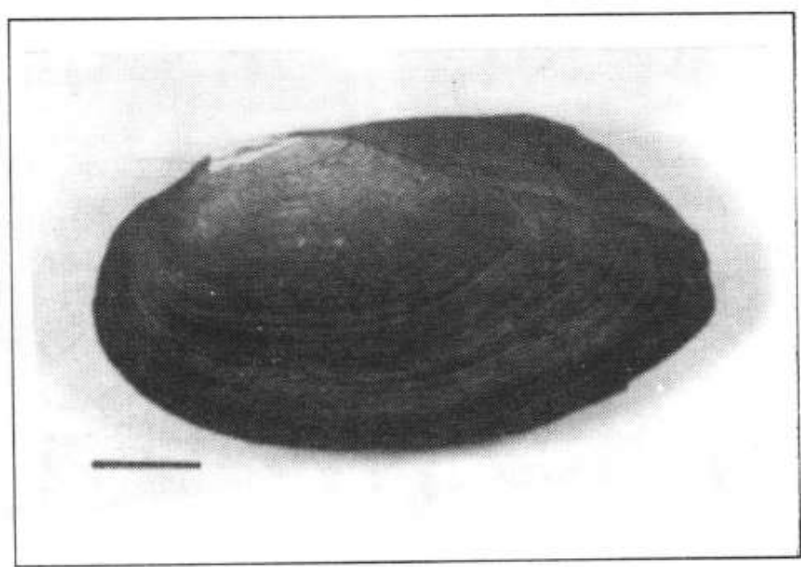

Fig. 7: Concha de Anodontites sp. Vista externa.

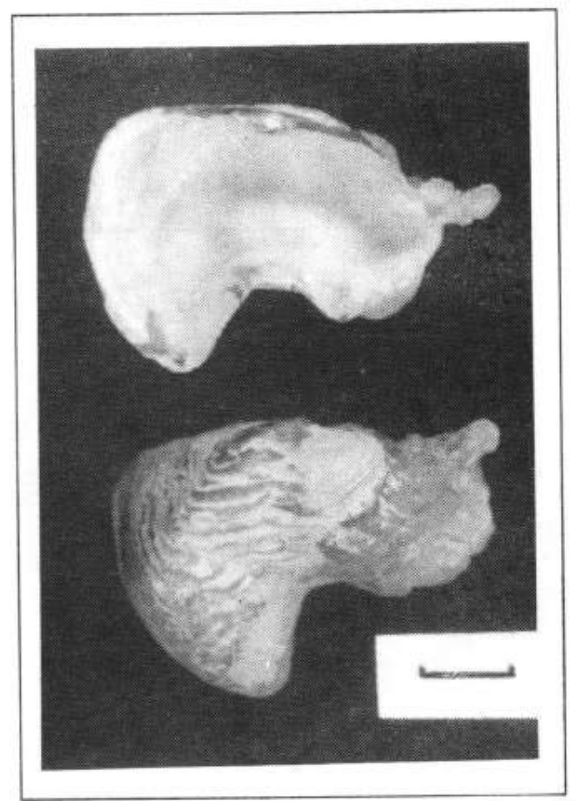

Fig. 9: Concha de Bartlettia stefanensis, (Moricand, 1856). Superior: vista interna, Inferior: vista externa. 


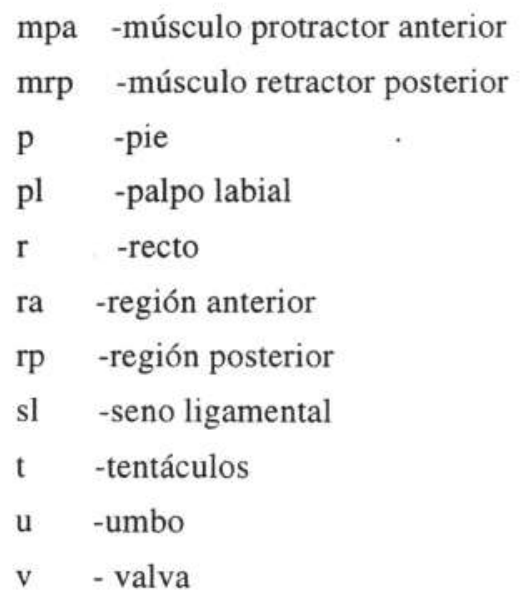

\section{REFERENCIAS BIBLIOGRAFICAS}

BONETTO, A.A. 1965. Almejas Sudamericanas de la Tribu Castaliini. Physis 25(69): 187- 196.

CASTELLANOS, Z \& LANDONI, N.1990. La Familia Mycetopodidae Gray, 1840 en la República Argentina 70 pp. En: Fauna de Agua Dulce de la República Argentina (ed. R. Ringuelet). Museo de La Plata. Facultad de Ciencias Naturales -UNLP.

MANSUR, M.C.D. 1972. Morfologia do sistema digestivo de Castalia undosa martensi (Ihering, 1891)(Bivalvia, Hyriidae). Iheringia Ser. Zool. 41:21-34.

MANSUR, M.C.D. Monocondylaea minuana Orb.,1835. Variabilidad da concha e morfologia do sistema digestivo (Bivalvia,Mycetopodidae). Iheringia Ser.Zool. 45:325

MANSUR, M.C \& OLIVEIRA DA SILVA, M.1990. Morfología e microanatomía comparada de Bartlettia stefanensis (MORICAND, 1856) e Anodontites tenebricosus (Lea, 1834) (Bivalvia, Unionoida, Muteloidea).Amazoniana 11 (2): 147-166

MANSUR, M.C \& VALER, R. M. 1992. Moluscos bivalves do Rio Uraricoera e Rio Branco, Roraima Brasil. Amazoniana 12 (1): 85 - 100.

PARODIZ, J.J. 1968. Annotated Catalogue of the genus Diplodon (UnionaceaHyriidae). Stekiana 30:1-30.

PARODIZ. J. J. 1982. Mollusca. En: Biota Acuática de Sudamérica Austral (Ed. por S. H. HURLBERT.) pp: 320 329. San Diego.

STRAYER, D.L. AND SMITH, L.C. 1993. Distribution of the Zebra Mussel (Dreissenia polymorpha) in Estuaries and Brackish Waters. Zebra Mussels: Biology, impacts, and control (Eds T.F Nalepa \& D.W. Schloesser), pp. 715727. Lewis Publishers, Ann Arbor, MI.

VEITENHEIMER, I \& MANSUR, M.C.D. 1978. Morfología, histología e ecología de Mycetopoda le gumen (Martens, 1888) (Bivalvia,Mycetopodidae)Iheringia Ser.Zool. 52:33-71.

VIVAR, R. P. HUAMAN; G. LAMAS; H. LARREA; L. PACHAS y M. OVIEDO. 1992. Moluscos de la región amazónica: Análisis sistemático preliminar. II Reunión Anual del ICBAR. U.N.M.S.M. Libro Resumen. pp.60.

WARREN, R. E. 1991. Freshwater Mussels as Paleoenvironmental Indicators: A Quantitative Approach to Assemblage Analysis. Illinois State Museum Scientific Papers. 23 (52): 23 - 66.

WARREN, R.E. 1995. Premodern Pleurobema (Rafinesque 1820) from the Illinois River. Transactions of the Illinois State Academy of Science 88.(in press). 